\title{
AN UPPER BOUND FOR HIGHER TOPOLOGICAL COMPLEXITY AND HIGHER STRONGLY EQUIVARIANT COMPLEXITY
}

\author{
AMIT KUMAR PAUL AND DEBASIS SEN
}

\begin{abstract}
We prove an upper bound of higher topological complexity $\mathrm{TC}_{n}(X)$ using higher $\mathcal{D}$-topological complexity $\operatorname{TC}_{n}^{\mathcal{D}}(X)$ of a space $X$. An intermediate invariant $\widetilde{\mathrm{TC}}_{n}(X)$ is used in the proof. We interpret this invariant $\widetilde{\mathrm{TC}}_{n}(X)$ as higher analogue of strongly equivariant topological complexity of the universal cover of $\widetilde{X}$ with the action of the fundamental group of $X$.
\end{abstract}

\section{INTRODUCTION}

The topological complexity $\mathrm{TC}(X)$ of a path connected space $X$ was introduced by Farber (see [10]). It is a measure of the complexity to construct a motion-planning algorithm on the space $X$. Let $I=[0,1]$ and $P X=X^{I}$ denotes the free path space. Consider the fibration

$$
p: P X \rightarrow X \times X, \gamma \mapsto(\gamma(0), \gamma(1)) .
$$

Then $\operatorname{TC}(X)$ is defined to be the least positive integer $k$ such that there exists an open cover $\left\{U_{1}, \cdots, U_{k}\right\}$ of $X \times X$ with continuous section of $p$ over each $U_{i}$ (i.e. a continuous map $s_{i}: U_{i} \rightarrow P X$ satisfying $\pi \circ s_{i}=\operatorname{Id}_{U_{i}}$ for $\left.i=1,2, \cdots, k\right)$. Generalising the idea, Rudyak defined higher topological complexity (see [18]). He introduced $n$-th topological complexity $\mathrm{TC}_{n}(X), n \geq 2$ such that $\mathrm{TC}_{2}(X)=\mathrm{TC}(X)$. We recall the definition of higher topological complexity in the next section. It is well known that $\mathrm{TC}_{n}(X)$ is homotopy invariaint. Therefore one can define topological complexity of a discrete group $\pi$ as $\mathrm{TC}_{n}(\pi)=\mathrm{TC}_{n}(K(\pi, 1))$, where $K(\pi, 1)$ is a Eilenberg- MacLane space with fundamental group $\pi$ and other homotopy groups trivial.

Computation of topological complexity is difficult. With a few known exact computations of these invariants, there has been work to get better bounds of these numbers. Using cohomological dimension $\operatorname{cd}(\pi)$ of the fundamental group $\pi=\pi_{1}(X)$, A. Costa and M. Farber ([2]) obtained the following upper bound for a finite cell complex $X$ :

$$
\mathrm{TC}(X) \leq 2 \operatorname{cd}(\pi)+\operatorname{dim} X+1 .
$$

Further we know $\operatorname{cd}(\pi)+1 \leq \mathrm{TC}(\pi) \leq 2 \mathrm{~cd}(\pi)+1$. In ([3]) A. Dranishnikov improves this an upper bound to $\mathrm{TC}(X) \leq \mathrm{TC}(\pi)+\operatorname{dim} X$. Later in $([12])$ the authors introduced a $\mathcal{D}$-topological complexity $\mathrm{TC}^{\mathcal{D}}(X)$ which has the property $\mathrm{TC}^{\mathcal{D}}(X) \leq \mathrm{TC}(\pi)$. They showed that $\mathrm{TC}(X) \leq \mathrm{TC}^{\mathcal{D}}(X)+\left\lceil\frac{2 \operatorname{dim} X-r}{r+1}\right\rceil$, where $r$ is the connectivity of the universal cover of $X$. Clearly this gives a better bound. We generalise the result for higher $\mathrm{TC}_{n}(X)$.

Date: December 13, 2019.

2010 Mathematics Subject Classification. Primary: 55M30 ; Secondary: 55R91.

Key words and phrases. Topological complexity, LS category, Equivariant topological complexity. 
Theorem A. Let $X$ be a finite dimensional simplicial complex such that its universal cover $\widetilde{X}$ is r-connecetd. Then we have,

$$
\mathrm{TC}_{n}(X) \leq \mathrm{TC}_{n}^{\mathcal{D}}(X)+\left\lceil\frac{n \operatorname{dim} X-r}{r+1}\right\rceil, n \geq 2 .
$$

(See Theorem 4.6).

In particular, for $r=1$, we obtain $\mathrm{TC}_{n}(X) \leq \mathrm{TC}_{n}(\pi)+\left\lceil\frac{n \operatorname{dim} X-1}{2}\right\rceil$ which a generalisation of [3, Theorem 3.3] of A. Dranishnikov (cf. Corollary [4.7) . He used strongly equivariant topological complexity to prove the result. Note that there are other versions of equivariaint topological complexity (cf. [7, 17, 19]) all of which differ slightly from each other. As in ([12]), to prove Theorem A an intermediate invariant $\widetilde{\mathrm{TC}}_{n}(X)$ is introduced. We introduce higher analogue of strongly equivariant complexity. Generalising [12, Proposition 3.8 ], we prove that $\widetilde{\mathrm{TC}}_{n}(X)$ can be viewed as the higher strongly equivariant complexity $\mathrm{TC}_{n, \pi}^{*}(\widetilde{X})$ of the universal cover $\widetilde{X}$ with the action of the fundamental group $\pi$.

Theorem B. For any finite simplicial complex $X$, we have

$$
\widetilde{\mathrm{TC}}_{n}(X)=\mathrm{TC}_{n, \pi}^{*}(\tilde{X}), n \geq 2,
$$

where $\tilde{X}$ be the universal covering and $\pi=\pi_{1}(X)$.

(See Theorem 5.12)

The upper bound of TC in [3] was deduced by showing that $\mathrm{TC}(E) \leq \mathrm{TC}(B)+$ TC $_{, G}^{*}(F)-1$ for a fiber bundle $E \rightarrow B$ with fiber $F$ and structure group $G$. We prove a similar result for higher topological complexity.

Theorem C. Let $E, B$ be two locally compact metric spaces and $E \rightarrow B$ be a fiber bundle with fiber $F$ and structure group $G$ acting properly on $F$. Then

$$
\mathrm{TC}_{n}(E) \leq \mathrm{TC}_{n}(B)+\mathrm{TC}_{n, G}^{*}(F)-1, n \geq 2 .
$$

(See Theroem 5.13, )

Organisation. The organisation of the rest of the paper is as follows: In Section 2, we recall some basic definitions related to topological complexity and LS category. In Section 3 we recall the definition of higher $\mathcal{D}$-topological complexity and prove some general properties. In section 4 , we introduce the invariant $\widetilde{\mathrm{TC}}_{n}(X)$ and use it to prove Theorem A. In the last section, we introduce higher strongly equivariant complexity, obtain its properties, and prove Theorem B, Theorem C.

1.1. Acknowledgements. The first author was supported by $\mathrm{PhD}$ research fellowship of Indian Institute of Technology, Kanpur.

\section{Preliminary}

Here we review basic concept of LS-category, topological complexity and higher topological complexity of a space $X$. We also recall some equivariant analogues. For details we refer to [5, 6, 7, 10, 14, 18, 16]. 
2.1. LS-category and topological complexity. Let $q: E \rightarrow B$ be a onto map, then the sectional category of $q$ is denoted by $\operatorname{secat}(q)$ and define as the minimal positive integer $k$ such that we have an open cover $\left\{U_{i}\right\}_{i=1}^{k}$ for $B$ and on each open subset $U_{i}$ we have a continuous map $s_{i}: U_{i} \rightarrow E$ with $q \circ s_{i}: U_{i} \rightarrow B$ is homotopic to the inclusion $\operatorname{Id}_{U_{i}}: U_{i} \hookrightarrow B$. The map $s_{i}$ is called local section for $q$. If $q: E \rightarrow B$ is fibration then $\operatorname{secat}(q)=\operatorname{genus}(q)$, where $\operatorname{genus}(q)$ of the fibration $q$ is the minimal positive integer $k$ such that we have an open cover $\left\{U_{i}\right\}_{i=1}^{k}$ for $B$ and on each open subset $U_{i}$ we have a continuous map $s_{i}: U_{i} \rightarrow E$ satisfying $q \circ s_{i}=\operatorname{Id}_{U_{i}}: U_{i} \hookrightarrow B$. We denote by $P_{0} X$ be the space of all paths in $X$ starts from some fix point (say $x_{0}$ ) and $P X=X^{I}$ be free path space of $X$. Consider the fibrations

$$
p_{0}: P_{0} X \rightarrow X, \gamma \rightarrow \gamma(1) ; p: P X \rightarrow X \times X, \alpha \rightarrow(\alpha(0), \alpha(1)) .
$$

2.2. Definition. The Lusternik-Schnirelmann category (LS-category) of $X$ is defined as cat $(X):=\operatorname{genus}\left(p_{0}\right)$. The topological complexity of $X$ is $\operatorname{TC}(X):=\operatorname{genus}(p)$.

For $n \geq 2$, let $I_{n}$ denotes the wedge of $n$ intervals $[0,1]_{j}, j=1,2, \cdots, n$, where $0_{j} \in[0,1]_{j}$ are identified. Consider the mapping space $X^{I_{n}}$ and the fibration

$$
e_{n}: X^{I_{n}} \rightarrow X^{n}, e_{n}(\alpha)=\left(\alpha\left(1_{1}\right), \alpha\left(1_{2}\right), \cdots, \alpha\left(1_{n}\right)\right) .
$$

The $n$-th topological complexity of $X$ is defined to be $\operatorname{TC}_{n}(X):=\operatorname{genus}\left(e_{n}\right)$. It can be defined alternatively as $\mathrm{TC}_{n}(X)=\operatorname{genus}\left(e_{n}^{\prime}\right)$, where

$$
e_{n}^{\prime}: X^{I} \rightarrow X^{n}, e_{n}^{\prime}(\alpha)=\left(\alpha(0), \alpha\left(\frac{1}{n-1}\right), \alpha\left(\frac{2}{n-1}\right), \cdots, \alpha(1)\right) .
$$

This is because $e_{n}$ and $e_{n}^{\prime}$ are both fibrational replacement of the diagonal map $X \rightarrow$ $X^{n}$. Clearly $\mathrm{TC}_{2}(X)$ is nothing but $\mathrm{TC}(X)$.

Topological complexity is closely related to LS-category, satisfying the relation

$$
\operatorname{cat}\left(X^{n-1}\right) \leq \mathrm{TC}_{n}(X) \leq \operatorname{cat}\left(X^{n}\right) \leq \mathrm{TC}_{n+1}(X) .
$$

It is clear from the above inequality that $\left\{\mathrm{TC}_{n}(X)\right\}$ is a non-decreasing sequence. If a space $Y$ is homotopy equivalent to $X$, then $\mathrm{TC}_{n}(Y)=\mathrm{TC}_{n}(X)$ for any $n \geq 2$. Consequently, $X$ is contractible if and only if $\mathrm{TC}_{n}(X)=1$ for any $n \geq 2$.

2.3. Equivariant LS-category. Throughout the paper, $G$ will denote a discrete group. A topological space $X$ with an action of a group $G$ is called a $G$-space. A continuous map $\phi: X \rightarrow Y$ between $G$-spaces is called a $G$-map (or an equivariant map) if $\phi(g x)=g \phi(x)$ for all $g \in G$ and $x \in X$. The set $\mathcal{O}(x)=\{g x ; g \in G\}$ is called the orbit of $x \in X$ and $G_{x}=\{g \in G ; g x=x\}$ is called the isotropy group at $x$. For a subgroup $H$ of $G$, the $H$-fixed point set of $X$ is given by

$$
X^{H}=\{x \in X ; h x=x \text { for all } h \in H\} .
$$

We call $X$ is $G$-connected if the $H$-fixed point set $X^{H}$ is path-connected for every subgroup $H$ of $G$. Let $Y$ be an another $G$-space and $\phi, \psi: X \rightarrow Y$ be two $G$-maps. Then $\phi$ is said to be $G$-homotopic to $\psi$, written as $\phi \simeq_{G} \psi$, if there is a $G$-map $F: X \times I \rightarrow Y$ with $F(x, 0)=\phi(x)$ and $F(x, 1)=\psi(x)$, where $G$ acts trivially on $I$ and diagonally on $X \times I$. Two $G$-spaces $X, Y$ are called $G$-homotopy equivalent if there are $G$-maps $\phi: X \rightarrow Y$ and $\phi^{\prime}: Y \rightarrow X$ such that $\phi \circ \phi^{\prime} \simeq_{G} \operatorname{Id}_{Y}$ and $\phi^{\prime} \circ \phi \simeq_{G} \operatorname{Id}_{X}$. 
A subset $U \subset X$ is called $G$-invariant if $g U \subseteq U$ for all $g \in G$. Such a $U \subseteq X$ is called $G$-categorical if there exists a $G$-homotopy $F: U \times I \rightarrow X$ such that $F(-, 0)$ is the inclusion map $U \hookrightarrow X$ and Image $(F(-, 1)) \subset \mathcal{O}\left(x^{\prime}\right)$ for some $x^{\prime} \in X$. We say $X$ is $G$-contractible if $X$ is $G$-categorical.

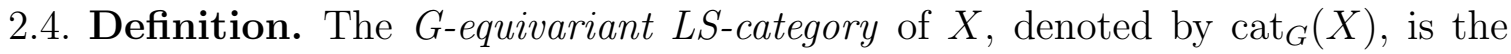
minimum positive integer $k$ such that $X$ can be covered by $k$ open sets $\left\{U_{1}, U_{2}, \cdots, U_{k}\right\}$, each of which is $G$-categorical.

Clearly $X$ is $G$-contractible if and only if $\operatorname{cat}_{G}(X)=1$. The $G$-equivariant category $\operatorname{cat}_{G}(X)$ is a $G$-homotopy invariant. Therefore if $X$ is $G$-homotopy equivalent to a point then $\operatorname{cat}_{G}(X)=1$, i.e. $X$ is $G$-contractible. The following lemma gives the converse implication.

2.5. Lemma. For a $G$-connected $G$-space $X$ with $X^{G} \neq \phi$, the $G$-contractibility of $X$ implies $X$ is $G$-homotopy equivalent to a point (in $X^{G}$ ).

Proof. Since $X$ is $G$-contractible, there is a $G$-homotopy $F_{t}^{\prime}: X \rightarrow X$ such that $F_{0}^{\prime}=\operatorname{Id}_{X}$ and $F_{1}^{\prime}(x) \in \mathcal{O}\left(x^{\prime}\right)$ for some $x^{\prime} \in X$. Consider an element $x_{0} \in X^{G}$ and the isotropy group $H=G_{x^{\prime}}$ at $x^{\prime}$. Then the both elements $x^{\prime}, x_{0} \in X^{H}$. Since $X$ is $G$ connected so $X^{H}$ is path-connected. Fix a path $\gamma: I \rightarrow X^{H}$, from $x^{\prime}$ to $x_{0}$. Note that $H \subseteq G_{\gamma(t)}$ for all $t \in I$. Define a homotopy $F_{t}^{\prime \prime}: \mathcal{O}\left(x^{\prime}\right) \rightarrow X$ by $F_{t}^{\prime \prime}\left(g \cdot x^{\prime}\right)=g \cdot \gamma(t)$, where $g \in G$. Then $F_{t}^{\prime \prime}$ is well defined and $F_{0}^{\prime \prime}=\operatorname{Id}_{\mathcal{O}\left(x^{\prime}\right)}, F_{1}^{\prime \prime}\left(g \cdot x^{\prime}\right)=x_{0}$. Define another homotopy $F_{t}=F_{t}^{\prime} * F_{t}^{\prime \prime}: X \rightarrow X$, where

$$
F_{t}^{\prime} * F_{t}^{\prime \prime}(x)= \begin{cases}F_{2 t}^{\prime}(x) & \text { if } 0 \leq t \leq \frac{1}{2} \\ F_{2 t-1}^{\prime \prime}\left(F_{1}^{\prime}(x)\right) & \text { if } \frac{1}{2} \leq t \leq 1,\end{cases}
$$

then $F_{t}$ is $G$-equivariant with $F_{0}=\operatorname{Id}_{X}$ and $F_{1}(x)=x_{0}$ for all $x \in X$.

2.6. Example. Let $\mathbb{Z}_{2}$-acts on $S^{n}, n \geq 2$ by reflection. Then $\operatorname{cat}_{\mathbb{Z}_{2}}\left(S^{n}\right)=2$. Assume the reflection keeps the hyperplane perpendicular to $x_{n}$ fixed. Take $U: x_{0}>-\frac{1}{2} \subset S^{n}$ and $V: x_{0}<\frac{1}{2} \subset S^{n}$. Then usual contraction of $U, V$ are equivariant. So $U, V$ are $\mathbb{Z}_{2}$-categorical hence $\operatorname{cat}_{\mathbb{Z}_{2}}\left(S^{n}\right) \leq 2$. If $\operatorname{cat}_{\mathbb{Z}_{2}}\left(S^{n}\right)=1$, then by Lemma $2.5 S^{n}$ is $\mathbb{Z}_{2^{-}}$ homotopy equivalent to a point, and in particular homotopy equivalent to a point which is not true. So $\operatorname{cat}_{\mathbb{Z}_{2}}\left(S^{n}\right)=2$.

We will need the following lemma in later section. We refer to [7, Theorem 3.16], [8, Theorem 2.23, Example 6.5], 9, Proposition 2.29] for the proof of the lemma.

2.7. Lemma. Suppose $Y_{i}$ is a $G_{i}$-space for $i=1,2$. Consider $Y_{1} \times Y_{2}$ as $G_{1} \times G_{2}$-space with the product action. If $Y_{1}^{G_{1}} \neq \phi$ and $Y_{2}^{G_{2}} \neq \phi$ then

$$
\operatorname{cat}_{G_{1} \times G_{2}}\left(Y_{1} \times Y_{2}\right) \leq \operatorname{cat}_{G_{1}}\left(Y_{1}\right)+\operatorname{cat}_{G_{2}}\left(Y_{2}\right)-1 .
$$

2.8. Deformable subset and $r$-cover. The topological complexity can be interpreted using deformable subsets. Let $A, U \subseteq X$. We call $U$ is $A$-deformable if there is a homotopy $h_{t}: U \rightarrow X$ with $h_{0}: U \hookrightarrow X$ is inclusion and $h_{1}(U) \subset A$. An open cover $\mathcal{C}=\left\{U_{1}, U_{2}, \cdots, U_{r}\right\}$ is called $A$-deformable if each $U_{i}$ is $A$-deformable. For a $G$-space $X$, let $A, U$ be invariant subsets. Then $U$ is called $A$-equivariantly deformable if the above homotopy is an equivariant homotopy. It is known that 
the topological complexity $\mathrm{TC}_{n}(X)$ of a space $X$ is the minimum number $k$ such that there is a $\Delta(X)$-deformable open cover of $X^{n}$ containing $k$ open sets, where $\Delta(X)=\left\{(x, x, \cdots, x) \in X^{n} ; x \in X\right\}$.

Now we recall some basic results about open covers which are described in ([3, 4, 15]). An open cover $\mathcal{C}=\left\{U_{1}, U_{2}, \cdots, U_{r+r^{\prime}}\right\}$ of a space $X$ is called $r$-cover if every subcollection of $r$ sets from $\mathcal{C}$ also a cover of $X$. We have the following simple observation.

2.9. Lemma. Let $\left\{U_{1}, U_{2}, \cdots, U_{r+r^{\prime}-1}\right\}$ be an $r$-cover and $\left\{V_{1}, V_{2}, \cdots, V_{r+r^{\prime}-1}\right\}$ be an $r^{\prime}$-cover of a space $X$, then $\left\{W_{1}, W_{2}, \cdots, W_{r+r^{\prime}-1}\right\}$ covers $X$ where $W_{i}=U_{i} \cap V_{i}$.

Proof. Let $x \in X$. By [3, Proposition 2.1] an open cover $\mathcal{C}=\left\{U_{1}, U_{2}, \cdots, U_{r+r^{\prime}-1}\right\}$ is an $r$-cover of a space $X$ if and only if each $x \in X$ is contained in at least $r^{\prime}$ sets of $\mathcal{C}$. Hence there is a subcollection $\left\{U_{i_{1}}, U_{i_{2}}, \cdots, U_{i_{r^{\prime}}}\right\}$ of $\left\{U_{1}, U_{2}, \cdots, U_{r+r^{\prime}-1}\right\}$ each of which contains $x$. Then the set $\left\{V_{i_{1}}, V_{i_{2}}, \cdots, V_{i_{r^{\prime}}}\right\}$ covers $X$. So $x \in V_{i_{k}}$ for some $k \in\left\{1,2, \cdots r^{\prime}\right\}$ and hence $x \in U_{i_{k}} \cap V_{i_{k}}=W_{i_{k}}$.

We will need the following result in last section.

2.10. Proposition. [3, Theorem 2.4] Assume $F$ is locally compact metric space and $A \subset F$. If $\left\{U_{i}^{\prime}\right\}_{i=1}^{r}$ is a A-deformable open cover of $F$, then for any $r^{\prime} \geq 0$ there is a A-deformable open $r$-cover $\left\{U_{j}\right\}_{j=1}^{r+r^{\prime}}$ of $F$ such that $U_{j}=U_{i}^{\prime}$ for $i=j \leq r$ and for $j>r, U_{j}=\sqcup_{i=1}^{r} V_{i}$ is a disjoint union with $V_{i} \subset U_{i}^{\prime}$.

If $G$ acts on $F$ and $A$ is $G$-invariant and $\left\{U_{i}^{\prime}\right\}_{i=1}^{r}$ is a A-equivariantly deformable open cover of $F$ by $G$-invariant sets, then for any $r^{\prime} \geq 0$ there is a A-equivariantly deformable open r-cover $\left\{U_{j}\right\}_{j=1}^{r+r^{\prime}}$ of $F$ by $G$-invariant sets such that $U_{j}=U_{i}^{\prime}$ for $i=j \leq r$ and for $j>r, U_{j}=\sqcup_{i=1}^{r} V_{i}$ is a disjoint union with $G$-invariant subsets $V_{i} \subset U_{i}^{\prime}$.

\section{Higher $\mathcal{D}$ - TOPOLOGiCAl COMPleXity}

In 13, Farber, Grant, Lupton and Oprea introduce $\mathcal{D}$-topological complexity for a path-connected space and proved that for a finite aspherical cell complex, the topological complexity and $\mathcal{D}$-topological complexity are same. In [11], Farber and Oprea define $n$-th $\mathcal{D}$-topological complexity $\operatorname{TC}_{n}^{\mathcal{D}}(X)$ and generalised the result. In [12], Farber, Grant, Lupton and Oprea proved some properties of $\mathcal{D}$-topological complexity. In this Section we generalised the results for $n$-th $\mathcal{D}$-topological complexity.

3.1. Definition. Let $X$ be a path-connected topological space with fundamental group $\pi=\pi_{1}\left(X, x_{0}\right)$. The $n$-th $\mathcal{D}$-topological complexity, $\operatorname{TC}_{n}^{\mathcal{D}}(X)$, is defined as the minimum number $k$ such that $X^{n}$ can be covered by $k$ open subsets, $X^{n}=$ $U_{1} \cup U_{2} \cup \cdots \cup U_{k}$, with the property that for any $i=1,2, \cdots, k$ and for every choice of the base point $u_{i} \in U_{i}$, the homomorphism $\pi_{1}\left(U_{i}, u_{i}\right) \rightarrow \pi_{1}\left(X^{n}, u_{i}\right)$ induced by the inclusion $U_{i} \rightarrow X^{n}$ takes values in a subgroup conjugate to the diagonal $\Delta \subset \pi^{n}$, where $\pi^{n}=\pi \times \pi \times \cdots \times \pi$ ( $n$-times).

We now interpret the above definition as sectional category of a certain covering map.

3.2. Proposition. Let $X$ be a path-connected, locally path-connected and semi-locally simply connected topological space with fundamental group $\pi=\pi_{1}\left(X, x_{0}\right)$. Let $q$ : 
$\widehat{X^{n}} \rightarrow X^{n}$ be the connected covering space corresponding to the diagonal subgroup $\Delta \subset \pi^{n}=\pi_{1}\left(X^{n}, X_{0}\right)$, where $X_{0}=\left(x_{0}, x_{0}, \cdots, x_{0}\right)$. Then

$$
\operatorname{TC}_{n}^{\mathcal{D}}(X)=\operatorname{secat}(q) \text {. }
$$

Proof. Let $\operatorname{secat}(q)=k$, with $\left\{U_{1}, U_{2}, \cdots, U_{k}\right\}$ be cover of $X^{n}$ and for each $i, s_{i}$ : $U_{i} \rightarrow \widehat{X^{n}}$ be a section on $U_{i}$ of $q$. Now by the lifting criterion of covering space $i_{*}\left(\pi_{1}\left(U_{i}, u_{i}\right)\right) \subseteq q_{*}\left(\pi_{1}\left(\widehat{X^{n}}, \widehat{x_{0}}\right)\right)\left(\right.$ where $\left.\widehat{x_{0}} \in \widehat{X^{n}}\right)$, that is, $i_{*}\left(\pi_{1}\left(U_{i}, u_{i}\right)\right) \subseteq q_{*}(\Delta)$. Hence $\operatorname{TC}_{n}^{\mathcal{D}}(X) \leq \operatorname{secat}(q)$.

Conversely, let $\mathrm{TC}_{n}^{\mathcal{D}}(X)=k$. Then $i_{*}\left(\pi_{1}\left(U_{i}, u_{i}\right)\right) \subseteq$ some conjugate of $\Delta$, means $i_{*}\left(\pi_{1}\left(U_{i}, u_{i}\right)\right) \subseteq q_{*}\left(\pi_{1}\left(\widehat{X^{n}}, \widehat{x_{0}}\right)\right)$. Again by lifting criterion of covering space, a lift $s_{i}: U_{i} \rightarrow \widehat{X^{n}}$ exist, i.e. section exist on $U_{i}$. Hence $\operatorname{TC}_{n}^{\mathcal{D}}(X) \geq \operatorname{secat}(q)$.

3.3. Example. For a path-connected space $X, \mathrm{TC}_{n}^{\mathcal{D}}(X)=1$ if and only if $X$ is simply connected. So we have $\operatorname{TC}_{n}^{\mathcal{D}}\left(S^{m}\right)=1$ for all $m, n \geq 2$.

To get an analogue of Equation (4) for $\mathcal{D}$-topological complexity, we recall the definition of Lusternik-Schnirelmann one-category.

3.4. Definition. Let $X$ be a path connected, locally path-connected and semi-locally simply connected space with universal cover $P: \widetilde{X} \rightarrow X$. The Lusternik-Schnirelmann one-category of $X$ is defined to be $\operatorname{cat}_{1}(X):=\operatorname{secat}(P)$ of $P$.

3.5. Proposition. If $X$ is a path-connected, locally path-connected and semi-locally simply connected topological space, then

$$
\operatorname{cat}_{1}\left(X^{n-1}\right) \leq \mathrm{TC}_{n}^{\mathcal{D}}(X) \leq \operatorname{cat}_{1}\left(X^{n}\right)
$$

Proof. Consider the commutative diagram,

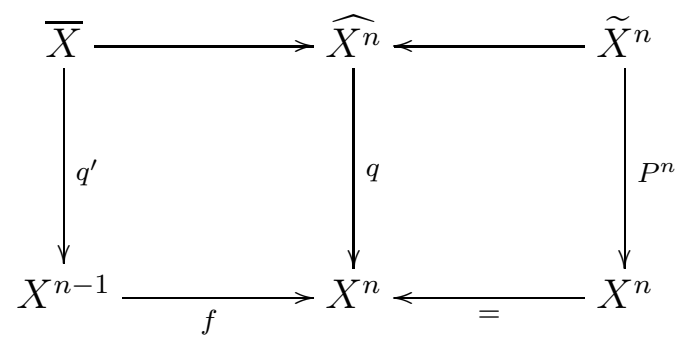

where $q: \widehat{X^{n}} \rightarrow X^{n}$ is the cover corresponds to the diagonal group $\Delta \subset \pi^{n}=$ $\pi \times \pi \times \cdots \times \pi$. The map $f: X^{n-1} \rightarrow X^{n}$ is the inclusion to the first $n-1$ factor, $f\left(x_{1}, x_{2}, \cdots, x_{n-1}\right)=\left(x_{1}, x_{2}, \cdots, x_{n-1}, *\right)$, where $* \in X$ is the base point and $\bar{X}$ is the preimage $q^{-1}\left(f\left(X^{n-1}\right)\right)$. Note that $q_{*}\left(\pi_{1}\left(\widehat{X^{n}}\right)\right)$ and $f_{*}\left(\pi_{1}\left(X^{n-1}\right)\right)$ spans $\pi_{1}\left(X^{n}\right)$. So using property of pullback covering by inclusion map we can say that $\bar{X}$ is covering space corresponding to the subgroup $f_{*}^{-1}\left(q_{*}\left(\pi_{1}\left(\widehat{X^{n}}\right)\right) \cap f_{*}\left(\pi_{1}\left(X^{n-1}\right)\right)\right)$ which is trivial. Thus $q^{\prime}: \bar{X} \rightarrow X^{n-1}$ is the universal cover of $X^{n-1}$.

Given an open subset $U \subset X^{n}$ with a section $s: U \rightarrow \widehat{X^{n}}$ we may restrict it to $f^{-1}(U) \subset X^{n-1}$ getting a section $s^{\prime}: f^{-1}(U) \rightarrow \bar{X}$. This shows that $\operatorname{cat}_{1}\left(X^{n-1}\right)=$ $\operatorname{secat}\left(q^{\prime}\right) \leq \operatorname{secat}(q)=\mathrm{TC}_{n}^{\mathcal{D}}(X)$, thus proving the first inequality. 
Next we consider the right square of the diagram. The map $P^{n}$ is the universal covering and hence secat $\left(P^{n}\right)=\operatorname{cat}_{1}\left(X^{n}\right) \geq \operatorname{secat}(q)=\mathrm{TC}_{n}^{\mathcal{D}}(X)$. This is the second inequality.

3.6. Corollary. For $X$ as above,

$$
\operatorname{cat}_{1}\left(X^{n-1}\right) \leq \mathrm{TC}_{n}^{\mathcal{D}}(X) \leq \operatorname{cat}_{1}\left(X^{n}\right) \leq \mathrm{TC}_{n+1}^{\mathcal{D}}(X) .
$$

In particular $\mathrm{TC}_{n}^{\mathcal{D}}(X) \leq \mathrm{TC}_{n+1}^{\mathcal{D}}(X)$, for all $n \geq 2$.

Now we relate higher topological complexity $\mathrm{TC}_{n}(X)$ with higher $\mathcal{D}$-topological complexity $\operatorname{TC}_{n}^{\mathcal{D}}(X)$.

3.7. Notation. Let $P: \widetilde{X} \rightarrow X$ be the universal cover of $X$. Let $\pi=\pi_{1}(X)$ denotes the fundamental group of $X$ and $\prod_{\pi} \tilde{X}$ stands for the quotient of $\widetilde{X}^{n}$ with respect to the diagonal action of $\pi$.

3.8. Proposition. For a path-connected, locally path-connected and semi-locally simply connected topological space $X$ one has $\mathrm{TC}_{n}^{\mathcal{D}}(X) \leq \mathrm{TC}_{n}(X)$.

Proof. Consider the projection $q: \prod_{\pi} \widetilde{X} \rightarrow X^{n}$. Clearly $q$ is a covering map with the property that the image of the induced homomorphism $q_{*}: \pi_{1}\left(\prod_{\pi} \widetilde{X}\right) \rightarrow \pi_{1}\left(X^{n}\right)$ is the diagonal. Hence by Proposition $3.2, \mathrm{TC}_{n}^{\mathcal{D}}(X)=\operatorname{secat}(q)$.

Now we define

$$
p: X^{I} \rightarrow \prod_{\pi} \widetilde{X}, \gamma \mapsto\left[\widetilde{\gamma}(0), \widetilde{\gamma}\left(\frac{1}{n-1}\right), \ldots, \widetilde{\gamma}\left(\frac{j}{n-1}\right), \cdots, \widetilde{\gamma}(1)\right],
$$

where $\widetilde{\gamma}: I \rightarrow \widetilde{X}$ is any lift of the path $\gamma: I \rightarrow X$ and the brackets $\left[x_{0}, x_{1}, \cdots, x_{n-1}\right]$ denote the orbit of the tuple $\left(x_{0}, x_{1}, \cdots, x_{n-1}\right) \in \widetilde{X}^{n}$ with respect to the diagonal action of $\pi$. The map $p$ is well-defined although the lift $\widetilde{\gamma}$ is not unique. We obtain the following commutative diagram.

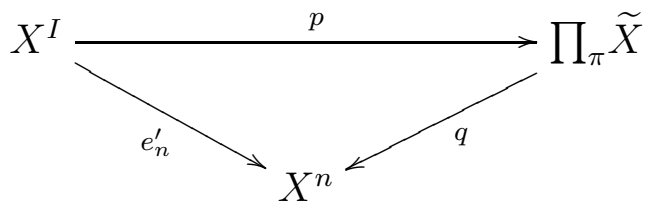

Clearly, a partial section $s: U \rightarrow X^{I}$ of $e_{n}^{\prime}$ gives a partial section $\widetilde{s}=p \circ s: U \rightarrow \prod_{\pi} \widetilde{X}$ of $q$. So we have $\operatorname{TC}_{n}^{\mathcal{D}}(X)=\operatorname{secat}(q) \leq \operatorname{secat}\left(e_{n}^{\prime}\right)=\operatorname{TC}_{n}(X)$.

For aspherical spaces, $\mathcal{D}$-topological complexity is same as topological complexity.

3.9. Lemma. [11, Lemma 4.2] Let $X$ be an aspherical $C W$ complex. Then

$$
\mathrm{TC}_{n}^{\mathcal{D}}(X)=\mathrm{TC}_{n}(X) \text {. }
$$

Now we show that the $\mathrm{TC}_{n}^{\mathcal{D}}(X)$ is also homotopy invariant.

3.10. Proposition. Assume that $f: X \rightarrow Y$ is a continuous map between pathconnected topological spaces such that the induced map $f_{*}: \pi_{1}(X) \rightarrow \pi_{1}(Y)$ is an isomorphism. Then we have,

$$
\mathrm{TC}_{n}^{\mathcal{D}}(X) \leq \mathrm{TC}_{n}^{\mathcal{D}}(Y)
$$


Proof. Let $U \subset Y^{n}$ be an open subset such that the induced homomorphism $\pi_{1}(U, u) \rightarrow$ $\pi_{1}\left(Y^{n}, u\right)$ takes values in a subgroup conjugate to the diagonal. Consider the preimage $V=(f \times f \times \cdots \times f)^{-1}(U) \subset X^{n}$. The map $\pi_{1}(V) \rightarrow \pi_{1}\left(X^{n}\right)$, induced by the inclusion, can be factored as the composition

$$
\pi_{1}(V) \rightarrow \pi_{1}(U) \rightarrow \pi_{1}\left(Y^{n}\right) \stackrel{\left(f_{*}^{-1}\right)^{n}}{\longrightarrow} \pi_{1}\left(X^{n}\right) .
$$

Since the second map takes values in a subgroup conjugate to the diagonal, hence the map $\pi_{1}(V) \rightarrow \pi_{1}\left(X^{n}\right)$ also has the same property. Therefore $\operatorname{TC}_{n}^{\mathcal{D}}(X) \leq \operatorname{TC}_{n}^{\mathcal{D}}(Y)$.

\subsection{Corollary. The higher D-topological complexities are homotopy invariant.}

Proof. Assume $f: X \rightarrow Y$ is a homotopy equivalence with inverse $g: Y \rightarrow X$. Then applying the above proposition to $f$ and $g$ we get $\operatorname{TC}_{n}^{\mathcal{D}}(X)=\mathrm{TC}_{n}^{\mathcal{D}}(Y)$.

Since $\mathrm{TC}_{n}^{\mathcal{D}}(X)$ is homotopy invariant, we can define $\mathrm{TC}_{n}^{\mathcal{D}}(\pi):=\mathrm{TC}_{n}^{\mathcal{D}}(K(\pi, 1))$ for a discrete group $\pi$. Note that $\mathrm{TC}_{n}^{\mathcal{D}}(\pi)=\mathrm{TC}_{n}(\pi)$ by Lemma 3.9.

3.12. Proposition. Let $X$ be a path-connected $C W$ complex with fundamental group $\pi=\pi_{1}(X)$. Then

$$
\mathrm{TC}_{n}^{\mathcal{D}}(X) \leq \mathrm{TC}_{n}^{\mathcal{D}}(\pi)
$$

Moreover, if there exist a positive integer $k \geq 2$ such that the homotopy groups $\pi_{j}(X)=0$ for all $j$ satisfying $1<j<k$ and $\pi$ has cohomological dimension $\leq k$, then

$$
\mathrm{TC}_{n}^{\mathcal{D}}(X)=\mathrm{TC}_{n}^{\mathcal{D}}(\pi) .
$$

Proof. The Eilenberg-Mac Lane complex $K=K(\pi, 1)$ can be constructed by attaching cells of dimension $\geq 3$ to $X$. Consider the inclusion map $i: X \hookrightarrow K$ which induces isomorphism of fundamental groups. So, using the Proposition 3.10 we can say that $\mathrm{TC}_{n}^{\mathcal{D}}(X) \leq \mathrm{TC}_{n}^{\mathcal{D}}(K)=\mathrm{TC}_{n}^{\mathcal{D}}(\pi)$.

For the second part, the Eilenberg-Mac Lane space $K=K(\pi, 1)$ can be obtained from $X$ by attaching cells of dimension $k+1, k+2, \cdots$. Now convert the inclusion $X \hookrightarrow K$ into a fibration with fiber $F$ satisfying $\pi_{i}(F)=\pi_{i+1}(K, X)$. Since $\pi_{1}(X) \simeq$ $\pi_{1}(K)$, we have $\pi_{i}(F)=\pi_{i+1}(K, X)=0$ for $i=0,1, \cdots, k-1$. The obstructions to finding a section of $X \rightarrow K$ lie in the groups $H^{i+1}\left(\pi, \pi_{i}(F)\right)=H^{i+1}\left(K, \pi_{i}(F)\right)$ and all these groups vanish because our computation with $\pi_{i}(F)$ and our assumption $\operatorname{cd}(\pi) \leq K$. Finally if we apply Proposition 3.10 to the section, which induces on the fundamental groups, we get $\mathrm{TC}_{n}^{\mathcal{D}}(X) \geq \mathrm{TC}_{n}^{\mathcal{D}}(K)=\mathrm{TC}_{n}^{\mathcal{D}}(\pi)$. So $\mathrm{TC}_{n}^{\mathcal{D}}(X)=\mathrm{TC}_{n}^{\mathcal{D}}(\pi)$.

We now show that if $X$ has a group structure, then the left side inequality of Corollary 3.6 is an equality.

3.13. Proposition. For any connected topological group $H, \mathrm{TC}_{n}^{\mathcal{D}}(H)=\operatorname{cat}_{1}\left(H^{n-1}\right)$.

Proof. Let $F: H^{n} \rightarrow H^{n-1}$ be the map given by the formula

$$
F\left(a_{1}, a_{2}, \cdots, a_{n}\right)=\left(a_{1} a_{n}^{-1}, a_{2} a_{n}^{-1}, \cdots, a_{n-1} a_{n}^{-1}\right) .
$$

Denote $\pi=\pi_{1}(H, e)$ and consider the induced map on fundamental groups 


$$
\phi=F_{*}: \pi^{n}=\pi_{1}\left(H^{n}, e^{n}\right) \longrightarrow \pi^{n-1}=\pi_{1}\left(H^{n-1}, e^{n-1}\right)
$$

where $\pi^{n}=\pi \times \pi \times \cdots \times \pi, n$-times and $e^{n}=(e, e, \cdots, e)$ is $n$-tuple in $H^{n}$. From the definition of $F$, it is clear that $F_{*}\left(\alpha_{1}, \alpha_{2}, \cdots, \alpha_{n}\right)=\left(\alpha_{1}-\alpha_{n}, \alpha_{1}-\alpha_{n}, \cdots, \alpha_{n-1}-\alpha_{n}\right)$. Note that the kernel of $\phi$ is $\Delta \subset \pi^{n}$, the diagonal subgroup. This gives a pullback diagram of covering maps

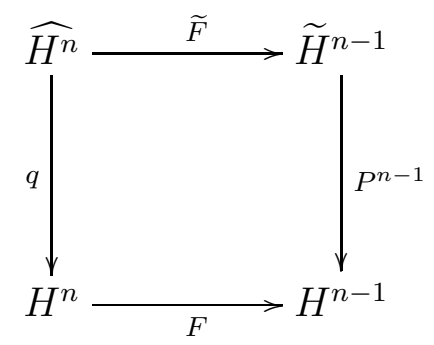

where $P: \widetilde{H} \rightarrow H$ is universal covering and $q$ is the covering corresponds to the diagonal subgroup. From the diagram we obtain $\operatorname{TC}_{n}^{\mathcal{D}}(H)=\operatorname{secat}(q) \leq \operatorname{secat}\left(P^{n-1}\right)=$ $\operatorname{cat}_{1}\left(H^{n-1}\right)$.

\section{The invariant $\widetilde{\mathrm{TC}}_{n}(X)$}

In this section we introduce an invaraint $\widetilde{\mathrm{TC}}_{n}(X)$ which is higher analogue of $\widetilde{\mathrm{TC}}(X)$ as defined in [12]. We use it to prove Theorem (A). Consider maps $E \stackrel{p}{\rightarrow}$ $\bar{X} \stackrel{q}{\rightarrow} X$, where $p$ is a fibration with fiber $F, q$ is a covering map with fiber $F_{0}$ and the space $\bar{X}$ is connected. The composition is a fibration with fiber $F^{\prime}$ which is homeomorphic to $F \times F_{0}$.

4.1. Definition. With notations as above, the number $\widetilde{\text { secat }}(E \stackrel{p}{\rightarrow} \bar{X} \stackrel{q}{\rightarrow} X)$ is the minimal integer $k \geq 1$ such that $X$ admits an open cover $X=U_{1} \cup U_{2} \cup \cdots \cup U_{k}$, with the property that for each $1 \leq i \leq k$, the fibration $p$ admits a continuous section over the open set $q^{-1}\left(U_{i}\right) \subset \bar{X}$.

It is clear from the definition that $\widetilde{\operatorname{secat}}(E \stackrel{p}{\rightarrow} \bar{X} \stackrel{q}{\rightarrow} X) \geq \operatorname{secat}(p)$ and $\widetilde{\operatorname{secat}}(E \stackrel{p}{\rightarrow}$ $\bar{X} \stackrel{q}{\rightarrow} X)=1$ if and only if $\operatorname{secat}(p)=1$. The following result is proved in [12, Proposition 3.2]. Note that we are counting from 1 in the definitions of genus and related things.

4.2. Lemma. With notations as above, we have

$$
\operatorname{secat}(q \circ p) \leq \operatorname{secat}(q)+\widetilde{\operatorname{secat}}(E \stackrel{p}{\rightarrow} \bar{X} \stackrel{q}{\rightarrow} X)-1 .
$$

Consider the quotient

$$
\left.E=\left\{\left(\omega, x_{1}, x_{2}, \cdots, x_{n}\right) ; \omega \in \tilde{X}^{I}, \omega(0)=x_{1}, \omega\left(\frac{1}{n-1}\right)=x_{2}, \cdots, \omega(1)=x_{n}\right)\right\} / \pi,
$$

where $\pi=\pi_{1}(X)$. Note that $E$ can be identified with $X^{I}$ by a choice of lift of path in $X$ to path in $\widetilde{X}$. The quotient by the fundamental group ensures that this is well defined. Recall that $\prod_{\pi} \widetilde{X}$ stands for the quotient of $\widetilde{X}^{n}$ with respect to the diagonal action of $\pi$ (see Notation 3.7). We define two maps $p, q$ as follows:

$$
p: E \rightarrow \prod_{\pi} \tilde{X}, p\left(\left[\omega, x_{1}, x_{2}, \cdots, x_{n}\right]\right) \mapsto\left[x_{1}, x_{2}, \cdots, x_{n}\right],
$$


and

$$
q: \prod_{\pi} \tilde{X} \rightarrow X^{n}, q\left(\left[x_{1}, x_{2}, \cdots, x_{n}\right]\right) \mapsto\left(P x_{1}, P x_{2}, \cdots, P x_{n}\right) .
$$

Here $P: \widetilde{X} \rightarrow X$ is the universal cover. Now we have the situation

$$
X^{I} \stackrel{p}{\longrightarrow} \prod_{\pi} \tilde{X} \longrightarrow X^{n} .
$$

For such $p, q$, it is clear that

$$
\operatorname{secat}(p \circ q)=\mathrm{TC}_{n}(X), \operatorname{secat}(q)=\mathrm{TC}_{n}^{\mathcal{D}}(X) .
$$

4.3. Definition. With notations as above, we define

$$
\widetilde{\mathrm{TC}}_{n}(X)=\widetilde{\operatorname{secat}}\left(X^{I} \stackrel{p}{\rightarrow} \prod_{\pi} \tilde{X} \stackrel{q}{\rightarrow} X^{n}\right)
$$

Applying the Lemma 4.2 in our this particular case we have

$$
\mathrm{TC}_{n}(X) \leq \mathrm{TC}_{n}^{\mathcal{D}}(X)+\widetilde{\mathrm{TC}_{n}}(X)-1
$$

4.4. Lemma. For a $C W$-complex $X$, the following statements are equivalent.

(i) For some $n \geq 2, \widetilde{\mathrm{TC}}_{n}(X)=1$.

(ii) The space $X$ is aspherical.

(iii) For all $n \geq 2, \widetilde{\mathrm{TC}}_{n}(X)=1$.

Proof. (i) $\Rightarrow$ (ii):

Suppose that $\widetilde{T C_{n}}(X)=1$, for some $n \geq 2$. Then the fibration $p: X^{I} \rightarrow \prod_{\pi} \tilde{X}$ has a continuous section. Now for $r \geq 2$ consider the composition

$$
\pi_{r}(X)=\pi_{r}\left(X^{I}\right) \stackrel{p_{*}}{\rightarrow} \pi_{r}\left(\prod_{\pi} \tilde{X}\right) \stackrel{\cong}{\rightarrow} \pi_{r}\left(X^{n}\right)=\bigoplus \pi_{r}(X),
$$

where $\bigoplus \pi_{r}(X)$ is the direct sum of $n$-copies of $\pi_{r}(X)$. Since $p$ has a section so this composition must surjective and it is possible only when $\pi_{r}(X)=0$, for all $r \geq 2$. So $X$ is aspherical.

(ii) $\Rightarrow$ (iii):

If $X$ is aspherical then $\widetilde{X}$ is contractible. The fiber of $p: X^{I} \rightarrow \prod_{\pi} \widetilde{X}$ is the mapping space $\operatorname{map}\left(\bigvee_{(n-1) \text { copies }} S^{1} \rightarrow \widetilde{X}\right)$, which is also contractible. This implies $p$ has a section and hence $\widetilde{\mathrm{TC}}_{n}(X)=1$ for all $n \geq 2$.

(iii) $\Rightarrow$ (i) is obvious.

4.5. Proposition. Let $Z=X \times Y$ where $X=K(\pi, 1)$ is aspherical and $Y$ is simply connected. Then $\mathrm{TC}_{n}^{\mathcal{D}}(Z)=\mathrm{TC}_{n}(X)$ and $\widetilde{\mathrm{TC}}_{n}(Z)=\mathrm{TC}_{n}(Y)$.

Proof. The first equality follows from the Proposition 3.10 applying on $X \rightarrow X \times$ $Y \rightarrow X$, injection and projection. The proof of second equality is similar as ([12], Proposition 3.11).

We now use the higher $\mathcal{D}$-topological complexity to give an upper bound of $\operatorname{TC}_{n}(X)$ using connectivity of the universal covering space $\widetilde{X}$ of $\mathrm{X}$. This is a generalisation of the result [12, Theorem 4.3]. 
4.6. Theorem. Let $X$ be a finite dimensional simplicial complex such that its universal cover $\widetilde{X}$ is r-connected. Then we have,

$$
\mathrm{TC}_{n}(X) \leq \mathrm{TC}_{n}^{\mathcal{D}}(X)+\left\lceil\frac{n \operatorname{dim} X-r}{r+1}\right\rceil
$$

In particular if $\widetilde{X}$ is $(n-1)$-connected, then

$$
\mathrm{TC}_{n}(X) \leq \mathrm{TC}_{n}^{\mathcal{D}}(X)+\operatorname{dim} X
$$

Proof. If we have a covering map $q: \bar{B} \rightarrow B$, with $B$ finite dimensional simplicial complex and a fibration $p: E \rightarrow \bar{B}$ with $(r-1)$-connected fiber for some $r \geq 0$, then by $([12$, Theroem 4.1$])$ we have

$$
\widetilde{\operatorname{secat}}(E \stackrel{p}{\rightarrow} \bar{B} \stackrel{q}{\rightarrow} B) \leq\left\lceil\frac{\operatorname{dim} B-r}{r+1}\right\rceil+1 .
$$

We apply this result to the defining maps $X^{I} \stackrel{p}{\rightarrow} \prod_{\pi} \widetilde{X} \stackrel{q}{\rightarrow} X^{n}$ of $\widetilde{\mathrm{TC}}_{n}(X)$. The fiber of the map $p$ is the mapping space $F=\operatorname{map}\left(\bigvee_{(n-1) \text { copies }} S^{1} \rightarrow \tilde{X}\right)$. Since $\tilde{X}$ is $r$-connected, we get that $F$ is $(r-1)$-connected. This can be seen by considering the fibration $P_{0} \widetilde{X} \rightarrow \widetilde{X}^{n}$ given by the projections at $0, \frac{1}{n-1}, \frac{2}{n-1}, \cdots, \frac{n-1}{n-1}=1$. This also has fiber $F$. Looking at the homotopy long exact sequence gives us the desired connectivity of $F$. So we get

$$
\widetilde{\mathrm{TC}}_{n}(X) \leq\left\lceil\frac{n \operatorname{dim} X-r}{r+1}\right\rceil+1 .
$$

Combining with the Equation (5) we get the Equation (6)

If $r=n-1$, then $\left\lceil\frac{n \operatorname{dim} X-r}{r+1}\right\rceil=\left\lceil\operatorname{dim} X-\frac{n-1}{n}\right\rceil=\operatorname{dim} X$, so we obtain

$$
\mathrm{TC}_{n}(X) \leq \mathrm{TC}_{n}^{\mathcal{D}}(X)+\operatorname{dim} X
$$

The following corollary is a generalisation of [3, Theorem 3.3].

4.7. Corollary. For a finite dimensional simplicial complex $X$ with fundamental group $\pi$ we have

$$
\mathrm{TC}_{n}(X) \leq \mathrm{TC}_{n}(\pi)+\left\lceil\frac{n \operatorname{dim} X-1}{2}\right\rceil .
$$

Proof. Combining Proposition 3.12 and Proposition 3.9 we have

$$
\mathrm{TC}_{n}^{\mathcal{D}}(X) \leq \mathrm{TC}_{n}^{\mathcal{D}}(\pi)=\mathrm{TC}_{n}(\pi)
$$

Since universal cover $\widetilde{X}$ is 1 -connected (simply connected), putting $r=1$ in the Equation (6) we get the result. 


\section{Higher STRONGLY EQUIVARIANT TOPOLOGICAL COMPLEXITY}

In ([7]) Colman and Grant introduced equivariant topological complexity for a $G$ space $Y$. It is denoted by $\mathrm{TC}_{G}(Y)$ and is defined as the minimum integer $k \geq 1$ such that there exist $G$-invariant open subsets $U_{1}, U_{2}, \cdots, U_{k}$ covering $Y \times Y$ under the diagonal action of $G$ on $Y \times Y$ and on each open subset there is $G$-equivariant section of the path fibration map $p: Y^{I} \rightarrow Y \times Y, \gamma \rightarrow(\gamma(0), \gamma(1))$. In ([9]) Bayeh and Sarkar generalized equivariant version to higher topological complexity. Dranishnikov ([3]) introduced strongly equivariant topological complexity $\operatorname{TC}_{G}^{*}(Y)$ of a $G$-space $Y$, in which the covering open subsets are $G \times G$ invariant and the sections $s_{i}: U_{i} \rightarrow Y^{I}$ are $G$-equivariant with diagonal action of $G$ on $U_{i}$. In this section we introduce higher strongly equivariant topological complexity $\mathrm{TC}_{n, G}^{*}(Y)$ of a $G$-space $Y$ and obtain some properties. After that we relate $\widetilde{\mathrm{TC}}_{n}(X)$ and $\mathrm{TC}_{n, \pi}^{*}(\widetilde{X})$, where $\tilde{X}$ is the universal cover of $X$ and $\pi=\pi_{1}(X)$.

5.1. Definition. For a $G$-space $Y$, consider $Y^{n}$ as a $G^{n}$-space with product action. Consider the fibration $e_{n}: Y^{I_{n}} \rightarrow Y^{n}$ (cf. Equation 2) for $Y$. The $G$-action on $Y$ naturally induces a $G$-action on $Y^{I_{n}}$. We define the $n$-th strongly equivariant topological complexity $\mathrm{TC}_{n, G}^{*}(Y)$, as the the minimal number $k$ such that $Y^{n}$ can be cover by $G^{n}$-invariant open sets $\left\{U_{i}: i=1, \cdots, k\right\}$ and there is a $G$-equivariant continuous section $s_{i}: U_{i} \rightarrow Y^{I_{n}}$ of $e_{n}$ for $i=1, \cdots, k$ (considering $G$ as the diagonal subgroup of $G^{n}$ ). If no such $k$ exist, then $\operatorname{TC}_{n, G}^{*}(Y)=\infty$.

The following lemma shows that we can take the fibration $e_{n}^{\prime}: Y^{I} \rightarrow Y^{n}$ in the above definition (cf. Equation 3). Later we will use them interchangeably.

5.2. Lemma. Let $Y$ be a $G$-space and $U$ be a $G^{n}$-invariant open set of $Y^{n}$, then admitting $G$-equivariant continuous section on $U$ of the maps $e_{n}: Y^{I_{n}} \rightarrow Y^{n}$ and $e_{n}^{\prime}: Y^{I} \rightarrow Y^{n}$ are equivalent.

Proof. Let $s: U \rightarrow Y^{I_{n}}$ be a $G$-equivariant continuous section of $e_{n}$. Define a map $\phi: I \rightarrow I_{n}$ by $\left[\frac{2 j-2}{2(n-1)}, \frac{2 j-1}{2(n-1)}\right]$ goes to linearly on $[0,1]_{j}$ in the reverse direction and $\left[\frac{2 j-1}{2(n-1)}, \frac{2 j}{2(n-1)}\right]$ goes to linearly on $[0,1]_{j+1}$ for $j=1,2, \cdots, n-1$. This map induces $\phi^{*}: Y^{I_{n}} \rightarrow Y^{I}$ such that the following diagram commutes.

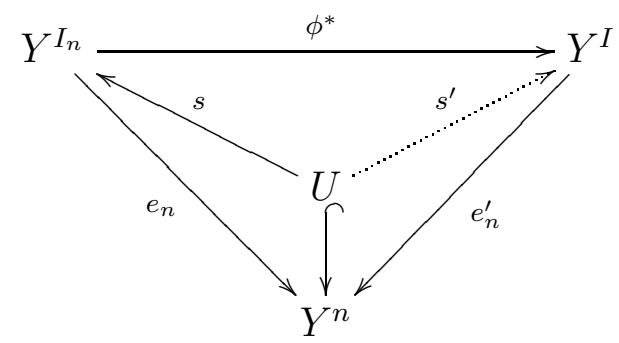


Consider the composition map $s^{\prime}=\phi^{*} \circ s: U \rightarrow Y^{I}$. Let $g \in G$ and $\left(y_{1}, y_{2}, \cdots, y_{n}\right) \in$ $U$. Using the fact $s: U \rightarrow Y^{I_{n}}$ is $G$-equivariant, we have,

$$
\begin{aligned}
\phi^{*} \circ s\left(g y_{1}, g y_{2}, \cdots, g y_{n}\right)(t) & =\phi^{*}\left(s\left(g y_{1}, g y_{2}, \cdots, g y_{n}\right)\right)(t) \\
& =s\left(g y_{1}, g y_{2}, \cdots, g y_{n}\right) \circ \phi(t) \\
& =g \cdot\left(s\left(y_{1}, y_{2}, \cdots, y_{n}\right)\right) \circ \phi(t) \\
& =g \cdot \phi^{*} \circ s\left(y_{1}, y_{2}, \cdots, y_{n}\right)(t) .
\end{aligned}
$$

So the map $s^{\prime}=\phi^{*} \circ s$ is a $G$-equivariant section of $e_{n}^{\prime}$.

Conversely, let $s^{\prime}: U \rightarrow Y^{I}$ be a $G$-equivariant continuous section of $e_{n}^{\prime}$. Define a map $\psi: I_{n} \rightarrow I$ by sending $[0,1]_{j}(1 \leq j \leq n)$ linearly to:

$$
\begin{array}{ll}
{\left[\frac{j-1}{n-1}, \frac{1}{2}\right] \text { in the reverse direction, }} & \text { if } j \leq \frac{n+1}{2} \\
{\left[\frac{1}{2}, \frac{j-1}{n-1}\right],} & \text { if } j>\frac{n+1}{2} .
\end{array}
$$

So the map $\psi$ induces $\psi^{*}: Y^{I} \rightarrow Y^{I_{n}}$ such that the following diagram commutes.

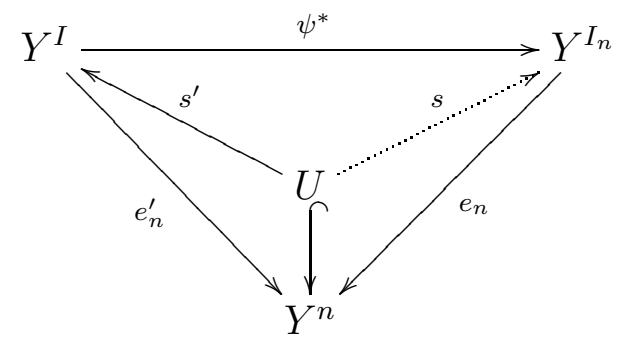

As in previous case the composition $s=\psi^{*} \circ s^{\prime}: U \rightarrow Y^{I_{n}}$ will be a $G$-equivariant section of $e_{n}$.

So in Definition 5.1 we can take the fibration $e_{n}^{\prime}$ instead of $e_{n}$. As in the other cases of higher topological complexity, the sequence $\left\{\mathrm{TC}_{n, G}^{*}(Y)\right\}_{n \geq 2}$ is non-decreasing.

5.3. Proposition. Let $Y$ be a $G$-space, then $\mathrm{TC}_{n+1, G}^{*}(Y) \geq \mathrm{TC}_{n, G}^{*}(Y)$, for any $n \geq 2$.

Proof. Take the inclusion $Y^{n} \rightarrow Y^{n+1}$ to the first $n$-factors. Given an open cover $\left\{U_{i}: i=1, \cdots, k\right\}$ of $Y^{n+1}$ by $G^{n+1}$-invariant subsets, take $V_{i}=U_{i} \cap Y^{n}$. Then $V_{i}$ are $G^{n}$ invariant with $G^{n} \hookrightarrow G^{n+1}$ as $\left(g_{1}, \cdots, g_{n}\right) \mapsto\left(g_{1}, \cdots, g_{n}, e\right)$. Restrict the section over $U_{i}$ to $V_{i}$. This satisfies the desired properties.

We now show that $n$-th strongly equivariant topological complexity is $G$-homotopy invariant.

5.4. Proposition. Let $X, Y$ be $G$-spaces and there are $G$-maps $\phi: X \rightarrow Y, \psi: Y \rightarrow$ $X$ such that $\phi \circ \psi \simeq_{G} \operatorname{Id}_{Y}$. Then $\mathrm{TC}_{n, G}^{*}(X) \geq \mathrm{TC}_{n, G}^{*}(Y)$. In particular if $X$ is $G$-homotopy equivalent to $Y$ then $\mathrm{TC}_{n, G}^{*}(X)=\mathrm{TC}_{n, G}^{*}(Y)$. 
Proof. Take an $G^{n}$-invariant open set $U \subset X^{n}$ with an equivariant section $s: U \rightarrow X^{I}$ of $e_{n}^{\prime}$. Consider $V=\left(\psi^{n}\right)^{-1}(U) \subset Y^{n}$. Then $V$ is also $G^{n}$-invariant.

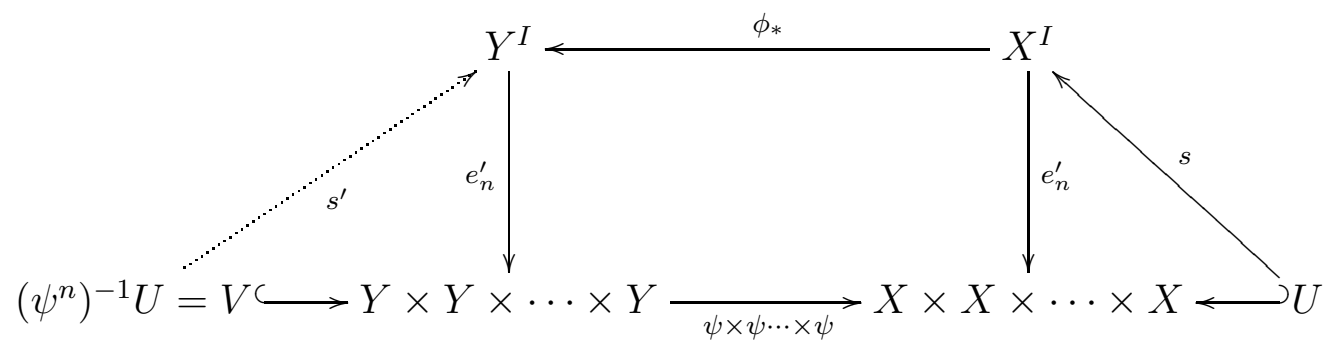

Define $s^{\prime}: V \rightarrow Y^{I}$ as $s^{\prime}\left(y_{1}, \cdots, y_{n}\right)=\phi_{*} \circ s \circ \psi^{n}\left(y_{1}, \cdots, y_{n}\right)$ where $\phi_{*}: X^{I} \rightarrow Y^{I}$ is the map induced by $\phi$. Then $e_{n}^{\prime} \circ s^{\prime}: V \rightarrow Y^{n}$ is the map $\left(y_{1}, \cdots, y_{n}\right) \mapsto(\phi \circ$ $\left.\psi\left(y_{1}\right), \cdots, \phi \circ \psi\left(y_{n}\right)\right)$ which is $G$-homotopic to identity.

5.5. Corollary. For a G-connected space $Y$ with $Y^{G} \neq \phi$, then $Y$ is $G$-contractible if and only if $\mathrm{TC}_{n, G}^{*}(Y)=1$, for some $n \geq 2$.

Proof. First assume that $Y$ is $G$-contractible. Since $Y$ is $G$-connected and $Y^{G} \neq \phi$, using Lemma 2.5 we can say that $Y$ is $G$-homotopy equivalent to a point. Hence the corollary follows from the above proposition.

Conversely, let $\mathrm{TC}_{n, G}^{*}(Y)=1$ for some $n \geq 2$. Then there is a $G$-equivariant section $s: Y^{n} \rightarrow Y^{I}$ of $e_{n}^{\prime}$. Fix $y_{0} \in Y^{G}$. Define a homotopy $H: Y \times I \rightarrow Y$ by $(y, t) \rightarrow s\left(y, y_{0}, y_{0}, \cdots y_{0}\right)(t)$. Clearly $H$ is a $G$-homotopy between identity map on $Y$ and $C_{y_{0}}$ (constant map on the orbit $\left.\mathcal{O}\left(y_{0}\right)\right)$. So $Y$ is $G$-contractible.

We now give some inequalities relating higher equivariant complexity $\mathrm{TC}_{n, G}(Y)$ of [9] and our $\mathrm{TC}_{n, G}^{*}(Y)$.

5.6. Proposition. Let $Y$ be a $G$-space. Then the following holds.

(a) For any $n \geq 2$, we have $\mathrm{TC}_{n, G}(Y) \leq \mathrm{TC}_{n, G}^{*}(Y)$.

(b) If $H$ and $K$ are subgroups of $G$ such that $Y^{H}$ is $K$-invariant, then

$$
\mathrm{TC}_{n, K}\left(Y^{H}\right) \leq \mathrm{TC}_{n, K}^{*}\left(Y^{H}\right) \leq \mathrm{TC}_{n, G}^{*}(Y) .
$$

In particular,

$$
\mathrm{TC}_{n}\left(Y^{H}\right) \leq \mathrm{TC}_{n, G}^{*}(Y), \mathrm{TC}_{n}(Y) \leq \mathrm{TC}_{n, K}(Y) \leq \mathrm{TC}_{n, G}^{*}(Y) .
$$

(c) If $Y$ is not $G$-connected, then $\mathrm{TC}_{n, G}^{*}(Y)=\infty$ for all $n \geq 2$.

Proof. (a) This simply follows from the definitions.

(b) Let us prove the inequality $\mathrm{TC}_{n, K}^{*}\left(Y^{H}\right) \leq \mathrm{TC}_{n, G}^{*}(Y)$. Let $U$ be an $G^{n}$-invariant open set of $Y^{n}$ and $s: U \rightarrow Y^{I}$ be a $G$-equivariant section for $e_{n}^{\prime}$. Define $V=U \cap\left(Y^{H}\right)^{n}$, is $K^{n}$-invariant as $U$ and $\left(Y^{H}\right)^{n}$ both are $K^{n}$-invariant. If we restrict the map $s$ on $V$, then for any $\left(y_{1}, \cdots, y_{n}\right) \in V$ and $h \in H$,

$$
h . s_{\left.\right|_{V}}\left(y_{1}, \cdots, y_{n}\right)=s_{\left.\right|_{V}}\left(h y_{1}, \cdots, h y_{n}\right)=s_{\left.\right|_{V}}\left(y_{1}, \cdots, y_{n}\right) \text {, }
$$

i.e. the path $s_{\left.\right|_{V}}\left(y_{1}, \cdots, y_{n}\right)$ is in $\left(Y^{H}\right)^{I}$. It is also clear that $s_{\left.\right|_{V}}$ is $K$-equivariant. So $s_{\left.\right|_{V}}: V \rightarrow\left(Y^{H}\right)^{I}$ is a $K$-equivariant section for $e_{\left.n\right|_{(Y H)}}^{\prime}:\left(Y^{H}\right)^{I} \rightarrow\left(Y^{H}\right)^{n}$. 
The others inequality follows from combining (a) with results of [9, Proposition 3.14].

(c) If $Y^{H}$ is not connected then, $\mathrm{TC}_{n}\left(Y^{H}\right)=\infty$. So this follows from part (b).

5.7. Remark. We can visualise the different inequalities of the above Proposition 5.6 the following picture, with an arrow goes from bigger to smaller number.

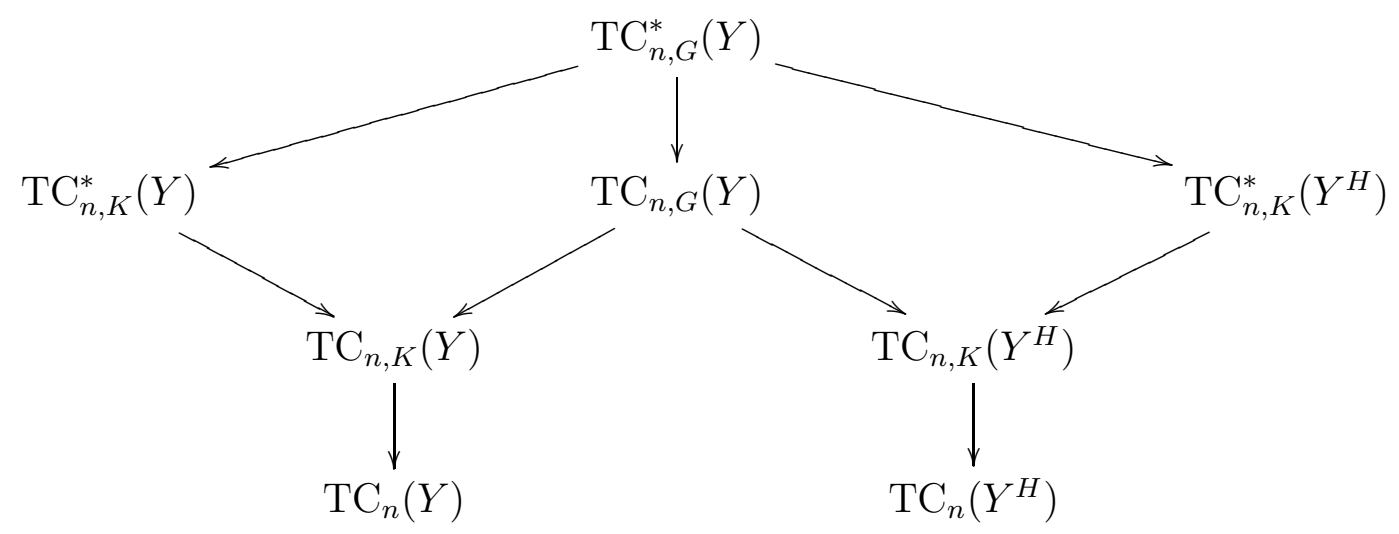

5.8. Lemma. We have $\mathrm{TC}_{n, G}^{*}(Y) \leq k$ if and only if there exist an open cover of $Y^{n}$ containing $k$ open sets such that each open set is $G^{n}$-invariant and $G$-equivariantly deformable into $\Delta(Y) \subset Y^{n}$.

Proof. It is enough to prove that, a $G$-equivariant section $s: U \rightarrow Y^{I_{n}}$ exist for $e_{n}: Y^{I_{n}} \rightarrow Y^{n}$ on some $G^{n}$-invariant open subset $U \subset Y^{n}$ if and only if there is a $G$-homotopy $H=\left(H_{1}, H_{2}, \cdots, H_{n}\right): U \times I \rightarrow Y^{n}$ with $H\left(\left(y_{1}, y_{2}, \cdots, y_{n}\right), 0\right) \in \Delta(Y)$ and $H\left(\left(y_{1}, y_{2}, \cdots, y_{n}\right), 1\right)=\left(y_{1}, y_{2}, \cdots, y_{n}\right)$. We set

$$
H_{j}\left(\left(y_{1}, \cdots, y_{j}, \cdots, y_{n}\right), t_{j}\right)=s\left(y_{1}, \cdots, y_{j}, \cdots, y_{n}\right)\left(t_{j}\right),
$$

where $t_{j} \in[0,1]_{j} \simeq I$ and $j=1, \cdots, n$. Then existence of one of $s$ and $H$ implies the other one. Hence the Lemma follows.

5.9. Proposition. Assume that $Y$ is a G-connected space with $Y^{G} \neq \phi$. Consider $Y^{m}$ with product $G^{m}$-action. Then

$$
\operatorname{cat}_{G^{n-1}}\left(Y^{n-1}\right) \leq \mathrm{TC}_{n, G}^{*}(Y) \leq \operatorname{cat}_{G^{n}}\left(Y^{n}\right) \leq n \operatorname{cat}_{G}(Y)-(n-1), n \geq 2 .
$$

Proof. For the first inequality, consider the pull-back square:

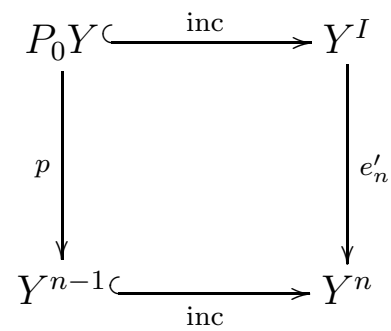

Here $P_{0} Y$ is the set of all paths in $Y$ starting at a base point $y_{0} \in Y^{G}$ and $Y^{n-1} \hookrightarrow$ $Y^{n}, y \mapsto\left(y_{0}, y\right)$. Then for a section of $e_{n}^{\prime}$ over $U \subseteq Y^{n}$, we get a section over $V=(\text { inc })^{-1} U \subseteq Y^{n-1}$. 
For the second inequality, take $U \subseteq Y^{n}$ be $G^{n}$-categorical with respect to product action. Then there is a $G^{n}$-homotopy $H: U \times I \rightarrow Y^{n}$ such that $H(a, 0)=a$ and $H(a, 1)=\left(y_{0}, \cdots, y_{0}\right)$ where $y_{0} \in Y^{G}$. Then $U$ is $G$-equivariantly deformable to $\Delta(Y)$. 2.7.

For the third inequality, we note that $\operatorname{cat}_{G^{n}}\left(Y^{n}\right) \leq n \operatorname{cat}_{G}(Y)-(n-1)$ by Lemma

5.10. Example. Let $\mathbb{Z}_{2}$ acts on $S^{m}$ by reflection. If $m=1$, then the fixed point set is disconnected. So in this case $\operatorname{TC}_{n, \mathbb{Z}_{2}}^{*}\left(S^{1}\right)=\infty$ for all $n \geq 2$. For $m \geq 2$, the fixed point set is $S^{m-1}$ which is path connected. By Example 2.6 $\operatorname{cat}_{\mathbb{Z}_{2}}\left(S^{m}\right)=2$. In this case

$$
\mathrm{TC}_{n, \mathbb{Z}_{2}}^{*}\left(S^{m}\right) \leq n \operatorname{cat}_{\mathbb{Z}_{2}}\left(S^{m}\right)-(n-1)=n .2-(n-1)=n+1 .
$$

Also from Proposition [5.6, we get

$$
\mathrm{TC}_{n, \mathbb{Z}_{2}}^{*}\left(S^{m}\right) \geq \mathrm{TC}_{n}\left(\left(S^{m}\right)^{\mathbb{Z}_{2}}\right)=\mathrm{TC}_{n}\left(S^{m-1}\right), \mathrm{TC}_{n, \mathbb{Z}_{2}}^{*}\left(S^{m}\right) \geq \mathrm{TC}_{n}\left(S^{m}\right)
$$

We know from [18, Section 4] that $\mathrm{TC}_{n}\left(S^{m}\right)=n$ if $m$ is odd and $\operatorname{TC}_{n}\left(S^{m}\right)=n+1$ if $m$ is even. Thus

$$
\mathrm{TC}_{n, \mathbb{Z}_{2}}^{*}\left(S^{m}\right)=n+1, \text { for all } m, n \geq 2 .
$$

Note that $\mathrm{TC}_{n}\left(S^{m} / \mathbb{Z}_{2}\right)=\mathrm{TC}_{n}\left(D^{m}\right)=1$.

5.11. Example. Let $S^{3}=\left\{\left.(z, w) \in \mathbb{C}^{2}|| z\right|^{2}+|w|^{2}=1\right\}$. Consider the $S^{1}$-action, defined by $\alpha .(z, w)=(\alpha z, w)$. Then the fixed point sets are $\left\{(0, w): w \in S^{1}\right\} \cong S^{1}$. So $\mathrm{TC}_{n, S^{1}}^{*}\left(S^{3}\right) \leq n \operatorname{cat}_{S^{1}}\left(S^{3}\right)-(n-1)$. But $\operatorname{cat}_{S^{1}}\left(S^{3}\right)=2$ (cf. [9, Example 3.20]). Therefore

$$
n \leq \mathrm{TC}_{n, S^{1}}^{*}\left(S^{3}\right) \leq n+1
$$

We have the following Theorem showing that the strongly equivarint complexity of the universal $\mathrm{TC}_{n, \pi}^{*}(\widetilde{X})$ is same as $\widetilde{\mathrm{TC}}_{n}(X)$ where $\pi$ is the fundamental group of $X$. This is a generalisation of ([12, Proposition 3.8]). The proof is similar.

5.12. Theorem. For any locally finite $C W$ complex $X$, we have

$$
\widetilde{\mathrm{TC}}_{n}(X)=\mathrm{TC}_{n, \pi}^{*}(\tilde{X})
$$

where $P: \widetilde{X} \rightarrow X$ be the universal covering and $\pi=\pi_{1}(X)$.

Proof. We first show $\widetilde{\mathrm{TC}}_{n}(X) \leq \mathrm{TC}_{n, \pi}^{*}(\widetilde{X})$. Let $e_{n}^{\prime}: \widetilde{X}^{I} \rightarrow \widetilde{X}^{n}$ be the map as above. Assume that $\widetilde{U} \subset \widetilde{X}^{n}$ be an $\pi^{n}$ invariant open set such that there is a $\pi$-equivariant section $\tilde{s}: \widetilde{U} \rightarrow \widetilde{X}^{I}$ of $e_{n}^{\prime}$. Consider the open set $V=\widetilde{U} / \pi \subset \prod_{\pi} \widetilde{X}$ where $\pi$-acts diagonally. We have the following commutative diagram. 


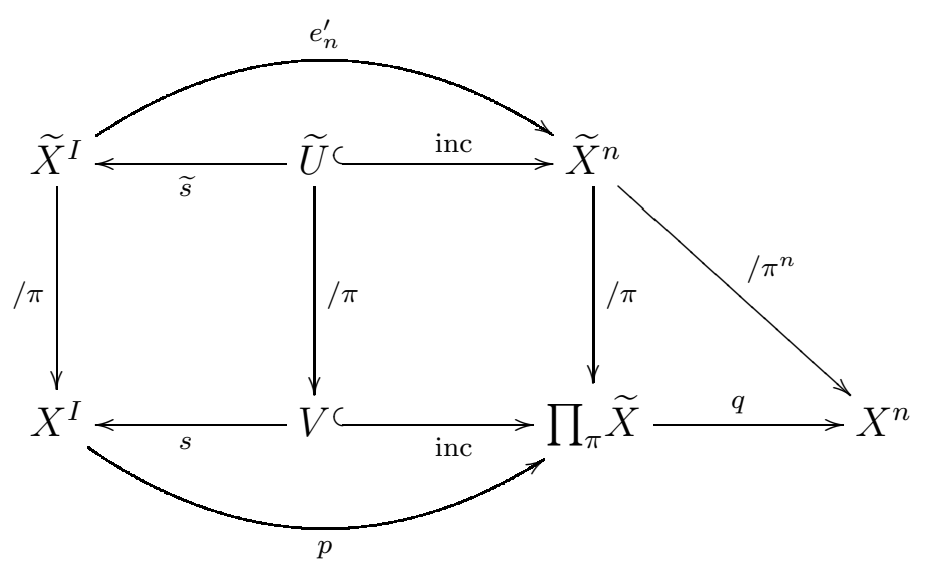

Since $\tilde{s}$ were $\pi$-equivariant, the section $s$ exists. Note that $V=q^{-1}\left(\widetilde{U} / \pi^{n}\right)$.

To prove the other inequality $\widetilde{\mathrm{TC}}_{n}(X) \geq \mathrm{TC}_{n, \pi}^{*}(\widetilde{X})$, it is enough to show that given a section $s$ as above, it can be lifted to a section $\widetilde{s}$. Since the $\pi$-action on the top rows are free, the vertical maps are principle $\pi$-bundles. Consider the classifying maps $\xi: X^{I} \rightarrow B \pi$ and $\xi^{\prime}: \prod_{\pi} \widetilde{X} \rightarrow B \pi$. Then the classifying map for the $\widetilde{U}$-bundle is $\xi^{\prime} \circ$ inc. The existence of $\widetilde{s}$ follows from the following fact of principal bundles: Let $E \rightarrow B$ and $E^{\prime} \rightarrow B^{\prime}$ be two principle $G$-bundles. Then a map $f: B^{\prime} \rightarrow B$ can be lifted to a bundle map $\tilde{f}: E^{\prime} \rightarrow E$ if and only if $\xi \circ f \simeq \xi^{\prime}$ where $\xi: B \rightarrow B G$ and $\xi^{\prime}: B^{\prime} \rightarrow B G$ are classifying maps of the respective principle $G$-bundles. We apply it to the $\pi$-bundles of the left square of the above diagram. The existence of the bundle map $e_{n}^{\prime}$ covering $p$ implies $\xi \simeq \xi^{\prime} \circ p$. Now note that $\xi \circ s \simeq \xi^{\prime} \circ p \circ s=\xi^{\prime}$. Therefore, by the above fact, the bundle map $\widetilde{s}$ exists making the diagram commutative.

We now use the strongly equivariant complexity to give an upper bound for higher complexity of total space of a fiber bundle. The following theorem is a generalisation of [3, Theorem 3.1].

5.13. Theorem. Let $E, B$ be two locally compact metric spaces and $p: E \rightarrow B$ be a fiber bundle with fiber $F$ and structure group $G$ acting properly on $F$. Then

$$
\mathrm{TC}_{n}(E) \leq \mathrm{TC}_{n}(B)+\mathrm{TC}_{n, G}^{*}(F)-1 .
$$

Proof. Let $\mathrm{TC}_{n}(B)=r, \mathrm{TC}_{n, G}^{*}(F)=r^{\prime}$ and $k=r+r^{\prime}-1$ such that $r, r^{\prime}, k \geq 1$. Consider $F^{n}$ as a $G$-space with diagonal action of $G$. Then there is a $\Delta(B)$-deformable open cover $\left\{U_{1}, U_{2}, \cdots, U_{r}\right\}$ of $B^{n}$ and a $G$-equivariantly deformable into $\Delta(F)$ open cover $\left\{V_{1}, V_{2}, \cdots, V_{r^{\prime}}\right\}$ of $F^{n}$ by $G^{n}$-invariant sets, by Lemma 5.8. Using Proposition 2.10 we can extend the above open covers to an $r$-cover $\left\{U_{1}, U_{2}, \cdots, U_{r}, \cdots, U_{k}\right\}$ and an $r^{\prime}$-cover $\left\{V_{1}, V_{2}, \cdots, V_{r^{\prime}}, \cdots, V_{k}\right\}$ for $B^{n}$ and $F^{n}$ respectively, with the same property.

Consider the universal $F^{n}$-bundle $q: F^{n} \times_{G^{n}} E\left(G^{n}\right) \rightarrow B\left(G^{n}\right)$ and classifying map $g: B^{n} \rightarrow B\left(G^{n}\right)$ for the $F^{n}$-bundle $p^{n}: E^{n} \rightarrow B^{n}$. Set $O_{i}=V_{i} \times_{G^{n}} E\left(G^{n}\right), i=$ $1,2, \cdots, k$ so that $\left\{O_{i}\right\}_{i=1}^{k}$ is an $r^{\prime}$-cover of $F^{n} \times_{G^{n}} E\left(G^{n}\right)$ and $W_{i}=O_{i}^{\prime} \cap\left(p^{n}\right)^{-1}\left(U_{i}\right)$, where $O_{i}^{\prime}=\left(g^{\prime}\right)^{-1}\left(O_{i}\right)$. Using Lemma 2.9 we can say that $\left\{W_{i}\right\}_{i=1}^{k}$ covers $E^{n}$. Now we show that the each set $W_{i}$ is deformable to $\Delta(E)$, in two steps. 
Step-I: Consider the composition map, $W_{i} \times I \stackrel{p^{n} \times \mathrm{Id}}{\longrightarrow} U_{i} \times I \stackrel{H}{\rightarrow} B^{n}$, where $H$ is a deformation of $U_{i}$ into $\Delta(B)$. Using the homotopy lifting property of the fibre bundle $f=p_{\left.\right|_{O_{i}^{\prime}} ^{n}}^{n}: O_{i}^{\prime} \rightarrow B^{n}$, we can say that $W_{i}$ can be deformed in $O_{i}^{\prime}$ to the preimage $f^{-1}(\Delta(B))$.

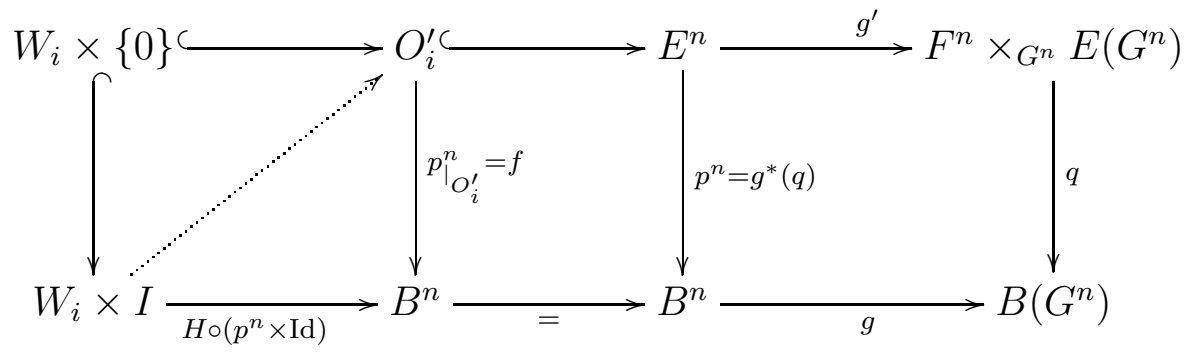

Step-II: Now we show that $f^{-1}(\Delta(B)) \subseteq O_{i}^{\prime}$ can be deformed into $\Delta(E)$. Let $\phi_{t}^{i}$ : $V_{i} \rightarrow F^{n}$ be an $G$-equivariant deformation of $V_{i}$ into $\Delta(F)$. It defines a deformation of $V_{i} \times{ }_{G} E G$ to $\Delta(F) \times{ }_{G} E G$ in $F^{n} \times{ }_{G} E G$. Observe that the bundle $q: F^{n} \times{ }_{G^{n}} E\left(G^{n}\right) \rightarrow$ $B\left(G^{n}\right)$ restricted over $\Delta(B G) \cong B G$ is $F^{n} \times_{G} E G \rightarrow B G$ with the diagonal action of $G$ on $F^{n}$. Then the above deformation defines a fiberwise deformation of $O_{i}$ over $\Delta(B G)$ into $\Delta\left(F \times \times_{G} E G\right)$. This will induce a fiberwise deformation of $O_{i}^{\prime}$ over $\Delta(B)$, i.e. of $f^{-1}(\Delta(B))$, into $\Delta(E)$.

The concatenation of the above two deformation in Step-I and Step-II defines a deformation of $W_{i}$ into $\Delta(E)$.

5.14. Remark. Following the arguments of [3, Theorem 3.3], the Corollary 4.7 can also be deduced using the above Theorem $[5.13$. Let $\widetilde{X}$ denote the universal cover of $X$. Consider the fiber bundle $\widetilde{X} \times_{\pi} E \pi \rightarrow B \pi$. It has fiber $\widetilde{X}$ and structure group $\pi$. Applying Theorem 5.13 to this bundle we get the following inequality

$$
\mathrm{TC}_{n}\left(\tilde{X} \times_{\pi} E \pi\right) \leq \mathrm{TC}_{n, \pi}^{*}(\tilde{X})+\mathrm{TC}_{n}(B \pi)-1 .
$$

Now by Theorem $5.12 \mathrm{TC}_{n, \pi}^{*}(\tilde{X})=\widetilde{\mathrm{TC}}_{n}(X)$. Also from the Equation [7, with $k=1$, we have $\widetilde{\mathrm{TC}}_{n}(X) \leq\left\lceil\frac{n \operatorname{dim} X-1}{2}\right\rceil+1$. Putting it in the above inequality

$$
\mathrm{TC}_{n}\left(\tilde{X} \times_{\pi} E \pi\right) \leq \mathrm{TC}_{n}(\pi)+\left\lceil\frac{n \operatorname{dim} X-1}{2}\right\rceil .
$$

Note that the map induced by covering projection $\widetilde{X} \times_{\pi} E \pi \rightarrow X$ is a homotopy equivalent, since it has contractible fiber $E \pi$. So $\mathrm{TC}_{n}\left(\widetilde{X} \times_{\pi} E \pi\right)=\mathrm{TC}_{n}(X)$.

\section{REFERENCES}

[1] A. ngel, H. Colman, "Equivariant topological complexities", Topological complexity and related topics, Contemp. Math., 702 (2018), pp. 115.

[2] A. Costa, M. Farber, "Motion planning in spaces with small fundamental groups", Commun. Contemp. Math., 12 (1) (2010), pp. 107119.

[3] A. Dranishnikov, "On topological complexity of twisted products", Topology Appl. 179 (2015), pp. 74- 80 . 
[4] A. N. Kolmogorov, "On the representation of continuous functions of many variables by superposition of continuous functions of one variable and addition", Amer. Math. Soc. Transl. (2) 28 (1963), pp. 5559.

[5] A. Švarc, "The genus of a fiber space", Amer. Math. Soc. Transl. Ser. 2, 55 (1966), pp. 49-140.

[6] H. Colman, "Equivariant LS-category for finite group actions", Lusternik-Schnirelmann category and related topics, Contemp. Math. 316, Amer. Math. Soc. (2002) pp. 35-40.

[7] H. Colman, M. Grant, "Equivariant topological complexity", Algebr. Geom. Topol. 12 (2012), no. 4, pp. 2299 - 2316.

[8] M. Bayeh, S. Sarkar, "Some aspects of equivariant LS-category", Topology Appl. 196 (2015), pp. 133- 154 .

[9] M. Bayeh, S. Sarkar, "Higher equivariant and invariant topological complexity", arXiv:1804.08006v1.

[10] M. Farber, "Topological complexity of motion planning", Discrete Comput. Geom. 29 (2003), no. 2, pp. 211-221.

[11] M. Farber, J. Oprea, "Higher topological complexity of aspherical spaces", Topology Appl. 258 (2019), pp. 142-160.

[12] M. Farber, M. Grant, G. Lupton, J. Oprea, "An upper bound for topological complexity", Topology Appl. 255 (2019), pp. 109 -125.

[13] M. Farber, M. Grant, G. Lupton, J. Oprea, "Bredon cohomology and robot motion planning", Algebr. Geom. Topol. 19 (2019), no. 4, pp. 2023 -2059.

[14] O. Cornea, G. Lupton, J. Oprea, D. Tanré, "Lusternik-Schnirelmann category, Math. Surveys and Monographs" 103, Amer. Math. Soc. (2003).

[15] P. A. Ostrand, "Dimension of metric spaces and Hilbert's problem 13", Bull. Amer. Math. Soc. 71 (1965), pp. $619-622$.

[16] W. Marzantowicz, "A G-Lusternik -Schnirelman category of space with an action of a compact Lie group", Topology 28 (1989) pp. 403-412.

[17] W. Lubawski, W. Marzantowicz, "Invariant topological complexity", Bull. London Math. Soc. 47 (2014), pp. 101-117.

[18] Y.B. Rudyak, "On higher analogs of topological complexity", Topology Appl. 157 (2010), no. 5, pp. 916-920.

[19] Z. Blaszczyk, M. Kaluba, "On equivariant and invariant topological complexity of smooth $\mathbb{Z}_{p^{-}}$ spheres", Proc. Amer. Math. Soc. 145(2017), pp. 4075 - 4086.

Department of Mathematics and Statistics, Indian Institute of Technology, KanPUR, UTtar PRADESh 208016, India

E-mail address: kamitp@iitk.ac.in, amitkrpaul23@gmail.com

Department of Mathematics and Statistics, Indian Institute of Technology, KanPUR, UTtAR PRADESH 208016, INDIA

E-mail address: debasis@iitk.ac.in 